JET snake magnetohydrodynamic equilibria

This article has been downloaded from IOPscience. Please scroll down to see the full text article.

2011 Nucl. Fusion 51072002

(http://iopscience.iop.org/0029-5515/51/7/072002)

View the table of contents for this issue, or go to the journal homepage for more

Download details:

IP Address: 128.178.125.164

The article was downloaded on 10/06/2011 at 07:29

Please note that terms and conditions apply. 


\title{
LETTER
}

\section{JET snake magnetohydrodynamic equilibria}

\author{
W.A. Cooper, J.P. Graves and O. Sauter \\ Ecole Polytechnique Fédérale de Lausanne (EPFL), Centre de Recherches en Physique des \\ Plasmas, Association Euratom-Confédération Suisse, CH1015 Lausanne, Switzerland \\ E-mail: wilfred.cooper@epfl.ch
}

Received 14 March 2011, accepted for publication 25 May 2011

Published 9 June 2011

Online at stacks.iop.org/NF/51/072002

\begin{abstract}
Magnetohydrodynamic (MHD) equilibrium states with a three-dimensional helical core that display the characteristics of a saturated ideal internal kink mode are computed to model snake structures that have been observed in the JET tokamak (Weller et al 1987 Phys. Rev. Lett. 59 2303). The equilibrium states are calculated with a peaked pressure profile and a weak to moderate reversed core magnetic shear with a minimum safety factor $q_{\text {min }}$ near unity in the neighbourhood of the mid-radius of the plasma. Snake equilibrium states are computed in the range $0.94<q_{\min }<1.03$. This range aligns with linearly unstable ideal MHD internal kink solutions of the purely axisymmetric branch of the equilibrium states. The energy difference between the bifurcated axisymmetric and helical snake equilibrium solutions is minimal. One very important novelty is that the helical structures are computed with an equilibrium code developed for three-dimensional (3D) stellarator applications in a tokamak context and cannot be obtained with standard Grad-Shafranov equation solvers.
\end{abstract}

(Some figures in this article are in colour only in the electronic version)

Snake structures have been reported in the JET tokamak with pellet injection [1] and also spontaneously due to central impurity accumulation [2]. The standard theoretical picture proposed for the formation of snakes is described by assuming that a pellet is ablated inside the $q=1$ rational magnetic island (or that impurities accumulate at this same place in the spontaneously generated snakes) [3]. We provide an alternative perspective. The pellet ablates on-axis, causing the plasma to cool locally. This displaces the plasma current channel radially outwards producing a hollow current profile with the minimum value of $q$ (the safety factor which corresponds to the inverse rotational transform) approaching unity off-axis. Under these conditions, the plasma becomes unstable to an ideal internal magnetohydrodynamic (MHD) kink mode which quickly saturates. The large density due to the pellet provides the allure of the snake. The spontaneous snake occurs because the impurities accumulate on axis that radiate cooling the plasma centre leading to similar conditions as with pellet injection. In this work, we demonstrate that three-dimensional (3D) snake equilibria exist in theory even with an imposed axisymmetric plasma boundary. It should be noted that it has been difficult to distinguish snake structures from multiple harmonic modes in JET experiments [4].
The application of stellarator equilibrium codes to tokamaks was pioneered by Garabedian where the NSTAB code obtained bifurcated solutions with local 3D structures near low-order rational surfaces that were interpreted as indicators of magnetic island formation [5]. The calculations we undertake are more global in character. We have previously applied the procedure to compute 3D helical equilibria to model TCV [6] and MAST [7] in which the sawteeth disappear, but continuous mode structures [8,9] and long-lived modes [10] remain in which we prescribe the plasma mass or pressure profles that are very flat in the plasma core as reported in these experiments. Saturated internal kinks have been reported in NSTX [11] and sawteeth change from kink-like to quasiinterchange modes in DIII-D as the plasma boundary is varied from oval to bean-shaped [12]. These phenomena could also be potentially described with the model we propose. ITER hybrid scenario equilibria with similar profiles are also predicted to develop 3D internal structures [13]. The snake equilibria, on the other hand, have more peaked pressure profiles.

The formulation of 3D MHD states is based on the minimization of the plasma energy $W$ in which the magnetic flux surfaces are constrained to be nested with a single 
magnetic axis. The static conditions can be described by

$$
W=\iiint \mathrm{d}^{3} x\left(\frac{B^{2}}{2 \mu_{0}}+\frac{p_{\|}(s, B)}{\Gamma-1}\right),
$$

where the magnetic field strength is $B, \mu_{0}=4 \pi \times 10^{-7} \mathrm{H} / \mathrm{m}$ is the permeability of free space, $p_{\|}$is the parallel pressure which is expressed as a function of the radial variable $s(0 \leqslant s \leqslant 1)$ and $B$, while $\Gamma$ is the adiabatic index. We solve the inverse equilibrium problem for the cylindrical coordinates given by the distance from the major axis $R=R(s, u, v)$ and the height off the mid-plane $Z=Z(s, u, v)$ where $0 \leqslant u \leqslant 2 \pi$ denotes the poloidal angle and $0 \leqslant v \leqslant 2 \pi / L$ is the toroidal angle with $L$ the number of field periods around the torus. The variation with respect to an artificial time variable $t$ yields

$$
\begin{aligned}
\frac{\mathrm{d} W}{\mathrm{~d} t} & =-\iiint \mathrm{d} s \mathrm{~d} u \mathrm{~d} v\left[F_{R} \frac{\partial R}{\partial t}+F_{Z} \frac{\partial Z}{\partial t}+F_{\lambda} \frac{\partial \lambda}{\partial t}\right] \\
& -\iint_{s=1} \mathrm{~d} u \mathrm{~d} v\left[R\left(p_{\perp}+\frac{B^{2}}{2 \mu_{0}}\right)\left(\frac{\partial R}{\partial u} \frac{\partial Z}{\partial t}-\frac{\partial Z}{\partial u} \frac{\partial R}{\partial t}\right)\right] .
\end{aligned}
$$

This can be solved by applying a steepest descent energy minimization scheme as described in the VMEC $[14,15]$ and ANIMEC [16] codes. The last integral in equation (2) corresponds to the motion of the plasma boundary. For the applications considered in this work, the plasma-vacuum interface is fixed, thus this term vanishes. The MHD forces are

$$
\begin{aligned}
F_{R}= & \frac{\partial}{\partial u}\left[\sigma \sqrt{g} B^{u}(\boldsymbol{B} \cdot \nabla R)\right]+\frac{\partial}{\partial v}\left[\sigma \sqrt{g} B^{v}(\boldsymbol{B} \cdot \nabla R)\right] \\
& -\frac{\partial}{\partial u}\left[R \frac{\partial Z}{\partial s}\left(p_{\perp}+\frac{B^{2}}{2 \mu_{0}}\right)\right]+\frac{\partial}{\partial s}\left[R \frac{\partial Z}{\partial u}\left(p_{\perp}+\frac{B^{2}}{2 \mu_{0}}\right)\right] \\
& +\frac{\sqrt{g}}{R}\left[\left(p_{\perp}+\frac{B^{2}}{2 \mu_{0}}\right)-\sigma R^{2}\left(B^{v}\right)^{2}\right],
\end{aligned}
$$$$
F_{Z}=\frac{\partial}{\partial u}\left[\sigma \sqrt{g} B^{u}(\boldsymbol{B} \cdot \nabla Z)\right]+\frac{\partial}{\partial v}\left[\sigma \sqrt{g} B^{v}(\boldsymbol{B} \cdot \nabla Z)\right]
$$$$
+\frac{\partial}{\partial u}\left[R \frac{\partial R}{\partial s}\left(p_{\perp}+\frac{B^{2}}{2 \mu_{0}}\right)\right]-\frac{\partial}{\partial s}\left[R \frac{\partial R}{\partial u}\left(p_{\perp}+\frac{B^{2}}{2 \mu_{0}}\right)\right],
$$

where $B^{u} \equiv \boldsymbol{B} \cdot \nabla u$ and $B^{v} \equiv \boldsymbol{B} \cdot \nabla v$. The $F_{\lambda}$ force corresponds to the binormal (normal to the field line on a flux surface) component of the momentum balance at the equilibrium state and is given by

$$
F_{\lambda}=\Phi^{\prime}(s)\left[\frac{\partial\left(\sigma B_{v}\right)}{\partial u}-\frac{\partial\left(\sigma B_{u}\right)}{\partial v}\right]
$$

where $\sigma>0$ is the firehose stability parameter [16, 17]. For the isotropic pressure conditions we address, $p_{\|}=p_{\perp}=p$ and $\sigma=1 / \mu_{0}$. Under these circumstances $p(s) \sim \mathcal{M}(s)\left[\Phi^{\prime}(s)\right]^{\Gamma}$ and we prescribe the plasma mass $\mathcal{M}$. The toroidal magnetic flux function is $\Phi$ and prime $\left(^{\prime}\right)$ indicates the derivative of a flux surface quantity with respect to $s$. Fourier decomposition in the angular variables $u$ and $v$ is applied and a special finite difference scheme is invoked for the radial discretization. Matrix preconditioning is used in the accelerated steepest descent scheme implemented. The flux surface averaged radial

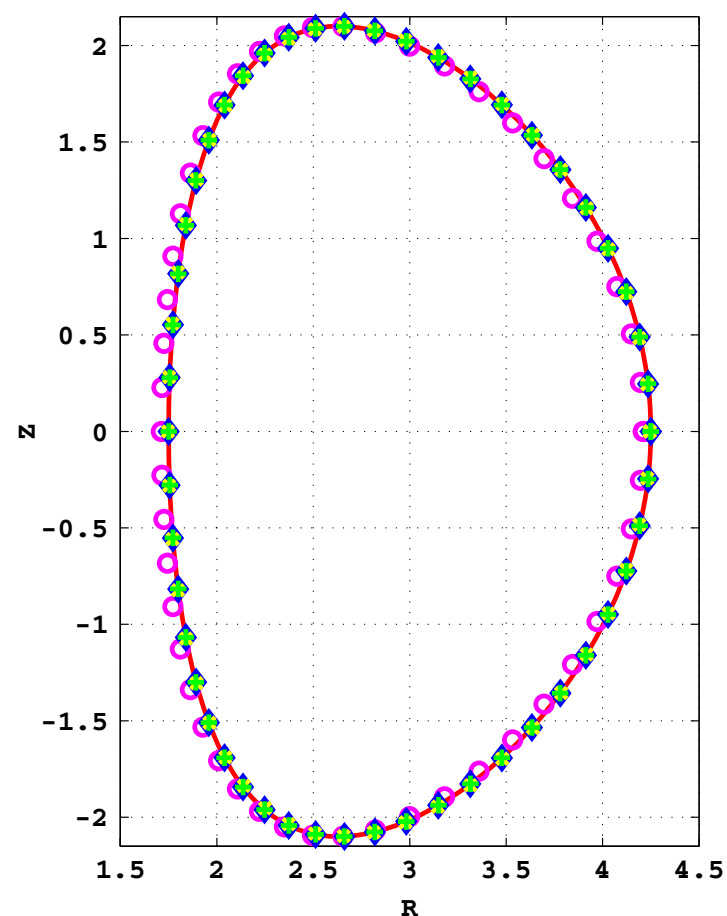

Figure 1. The JET plasma boundary shape according to the formula $R_{b}=R_{0}+a \cos (u+\delta \sin u) ; Z_{\mathrm{b}}=E a \sin u$ with $R_{0}=2.96 \mathrm{~m}$, $a=1.25 \mathrm{~m}, E=1.68$ and $\delta=0.3$ (solid line). A Fourier decomposition is given by the + symbols. The $o$ symbols correspond to a truncated Fourier set useful for analytic purposes.

force balance constitutes a diagnostic of the accuracy of the equilibrium state that is achieved which is given by

$\left\langle\frac{F_{s}}{\Phi^{\prime}(s)}\right\rangle=-\left\langle\left.\frac{1}{\Phi^{\prime}(s)} \frac{\partial p_{\|}}{\partial s}\right|_{B}\right\rangle-\frac{\partial}{\partial s}\left\langle\frac{\sigma B_{v}}{\sqrt{g}}\right\rangle-\iota(s) \frac{\partial}{\partial s}\left\langle\frac{\sigma B_{u}}{\sqrt{g}}\right\rangle$,

where

$$
\langle A\rangle=\frac{L}{(2 \pi)^{2}} \int_{0}^{2 \pi / L} \mathrm{~d} v \int_{0}^{2 \pi} \mathrm{d} u \sqrt{g} A(s, u, v) .
$$

In our investigations, the field period number $L$ is unity. In an axisymmetric or helically symmetric configuration, equation (6) corresponds to the flux surface averaged GradShafranov equation.

The equilibrium computation technique described has been implemented in the ANIMEC code [16], an anisotropic pressure version of the free boundary VMEC code $[14,15]$. The JET plasma boundary is presented in figure 1. A Fourier decomposition of this boundary yields amplitudes presented in table 1.

The JET snake equilibria that have been reported in $[1,2]$ occur in the range of toroidal magnetic field $B_{\mathrm{t}}=2-3.1 \mathrm{~T}$, and toroidal plasma current $I_{\mathrm{t}}=3-4.2 \mathrm{MA}$. We prescribe plasma mass and toroidal current profiles for an equilibrium within these ranges, specifically $I_{\mathrm{t}}=3.85 \mathrm{MA}, B_{\mathrm{t}}=3.1 \mathrm{~T}$ and volume averaged $\langle\beta\rangle=2.3 \%$. The pressure and inverse rotational transform $q$-profiles are shown in figures 2 and 3 , respectively. The minimum value of $q=1.0004$ is located at $\sqrt{s} \simeq 0.4544$. The details of the pressure profile do not appear to be important for theoretical simulations 
Table 1. The Fourier amplitudes that describe a JET tokamak plasma boundary.

\begin{tabular}{llll}
\hline$m$ & $n$ & $R_{m n}$ & $Z_{m n}$ \\
\hline 0 & 0 & 2.8654 & 0 \\
1 & 0 & 1.2498 & 2.0929 \\
2 & 0 & 0.31068 & -0.0776 \\
3 & 0 & $-7.3391 \times 10^{-4}$ & $5.2058 \times 10^{-4}$ \\
4 & 0 & $4.2409 \times 10^{-3}$ & $6.8633 \times 10^{-3}$ \\
5 & 0 & $8.4867 \times 10^{-4}$ & $-3.4499 \times 10^{-4}$ \\
6 & 0 & $-4.4441 \times 10^{-4}$ & $-5.7123 \times 10^{-4}$ \\
7 & 0 & $5.4061 \times 10^{-5}$ & $3.0482 \times 10^{-4}$ \\
8 & 0 & $8.5672 \times 10^{-5}$ & $6.3099 \times 10^{-5}$ \\
\hline
\end{tabular}

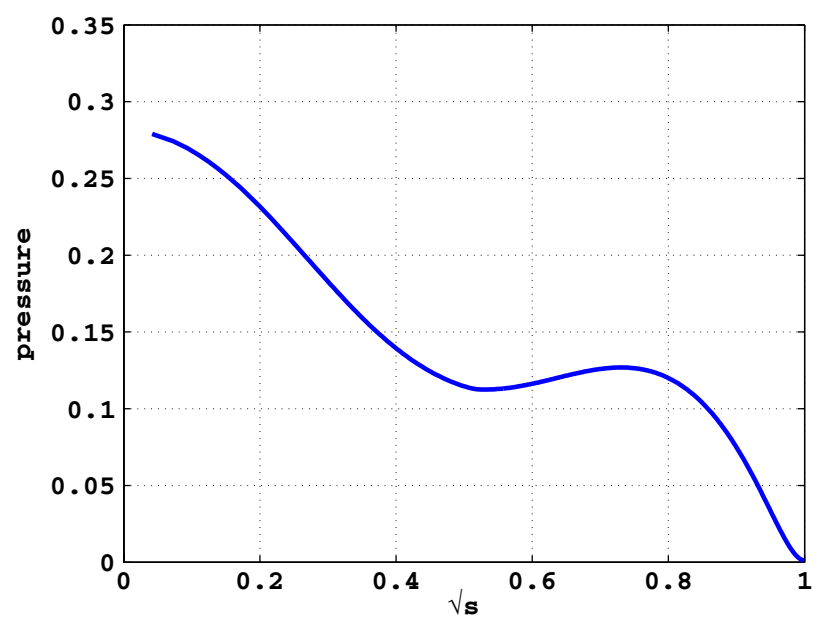

Figure 2. The pressure profile for JET snake-like equilibrium calculations as a function of $\sqrt{s}$.

of the snakes. However, the pressure profile applied has an $H$-mode type pedestal because flat extended low shear $q$-profiles, prevalent in this mode of tokamak operation, may predispose the development of the snake in contrast with the more conventional $q$-profiles of $L$-mode discharges.

MHD equilibrium computations using ANIMEC that generate states with a 3D helical core structure with the characteristics of the snake observed in JET usually require more than 10000 iterations to achieve convergence. The volume averaged horizontal force $F_{R}$ as a function of the number of iterations is displayed in figure 4 . The preconditioner is activated after 42000 iterations. Following an initial jump, the magnitude of $F_{R}$ drops to machine-like precision levels. If the preconditioner is turned on earlier in the run, the solution will either revert to the axisymmetric branch or it will oscillate between the axisymmetric and helical branches without converging. The number of radial grid points in our calculations is $N_{r}=289$. We would anticipate that the normalized radial force balance, extracted from equation (6) as a diagnostic for the equilibrium state, should achieve a level of $10^{-5}$ for quadratic convergence. With the matrix preconditioner active, the convergence is nearly quartic (with force residual levels below $10^{-8}$ ) except at $\sqrt{s} \sim 0.55$ and at the edge where it is closer to cubic.

The contours of constant pressure at four cross sections that encompass half a toroidal transit are shown in figure 5. The helical core internal structure is visible, while the edge region of the plasma retains axisymmetric character. The internal

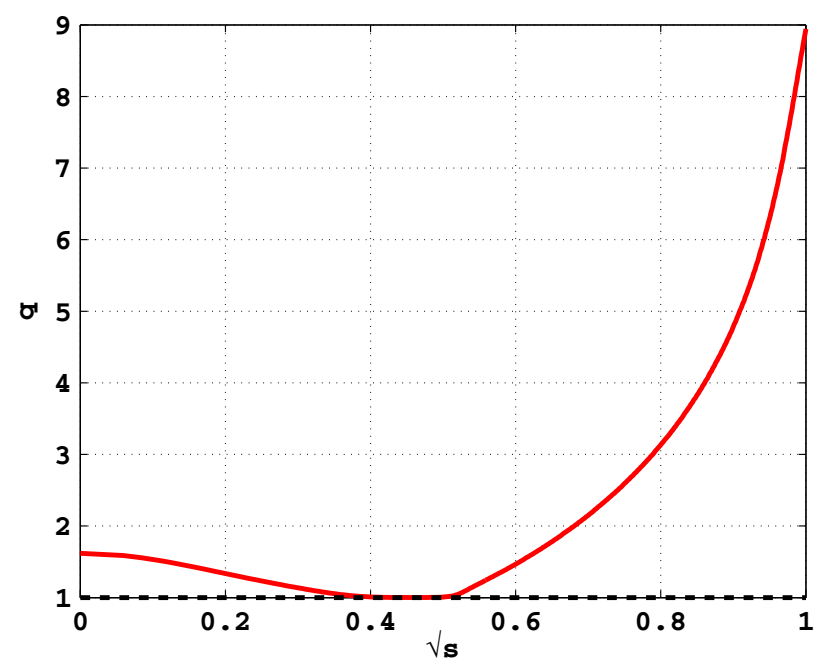

Figure 3. The $q$-profile for JET snake-like equilibrium calculations as a function of $\sqrt{s}$.

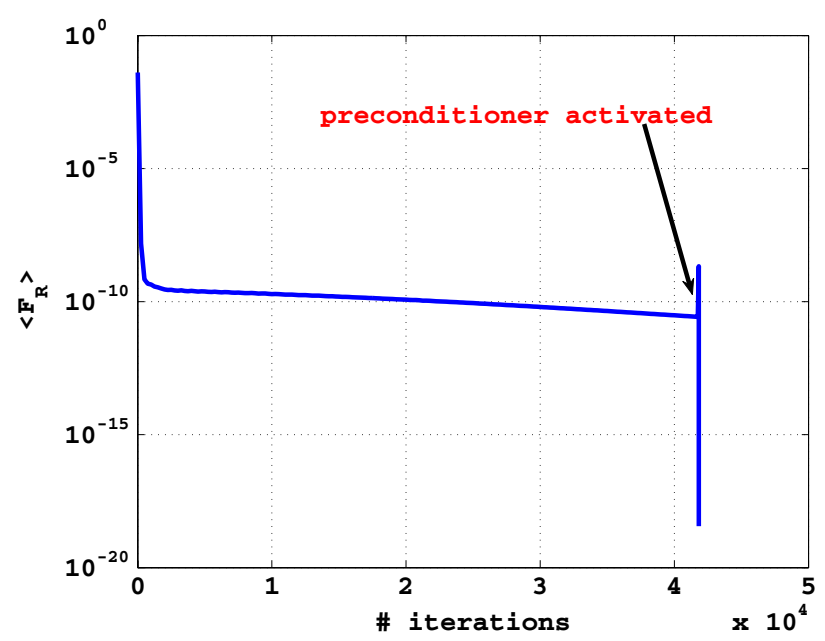

Figure 4. The volume averaged horizontal force $F_{R}$ evolution with the number of iterations.

plasma energy functional $\mu_{0} p+B^{2} / 2$ on the same four cross sections is displayed in figure 6 and shows that its magnitude scales roughly as the inverse distance from the major axis squared. This indicates that the internal helical flux surface structure does not alter the mod- $B$ lines which are very similar from one toroidal cross section to the next. This is expected at low $\langle\beta\rangle \sim 2 \%$. An alteration of the mod- $B$ contours would arise primarily from the diamagnetic effect of the (shifted) pressure on the magnetic field.

The equilibria that we calculate are static. In the experiment, the snake structure was detected due to its slow toroidal rotation. In figure 7 , we present a $3 \mathrm{D}$ graphic of the normalized pressure as a function of the distance from the major axis $R$ and the toroidal angle which is similar to the standard view of the snake (e.g. compare with figure 1 of [1] or [2]). The toroidal angle plays the role of time and the pressure that of the emissivity in the experimental description $[1,2]$.

We vary the toroidal current from 3.75 to $4.09 \mathrm{MA}$ (keeping its profile fixed) to test the sensitivity of the snake structures we compute with respect to the value of $q_{\text {min }}$. This 

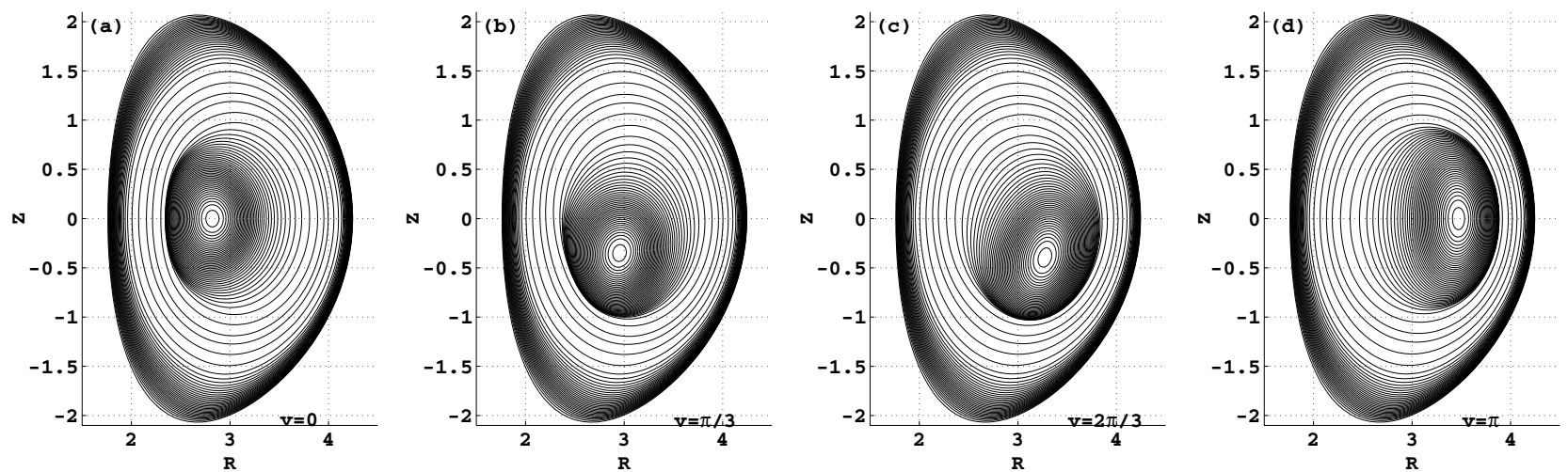

Figure 5. The contours of constant pressure at four toroidal cross sections that span half the way around the torus at toroidal angles $v=0$, $v=\pi / 3, v=2 \pi / 3$ and $v=\pi$.
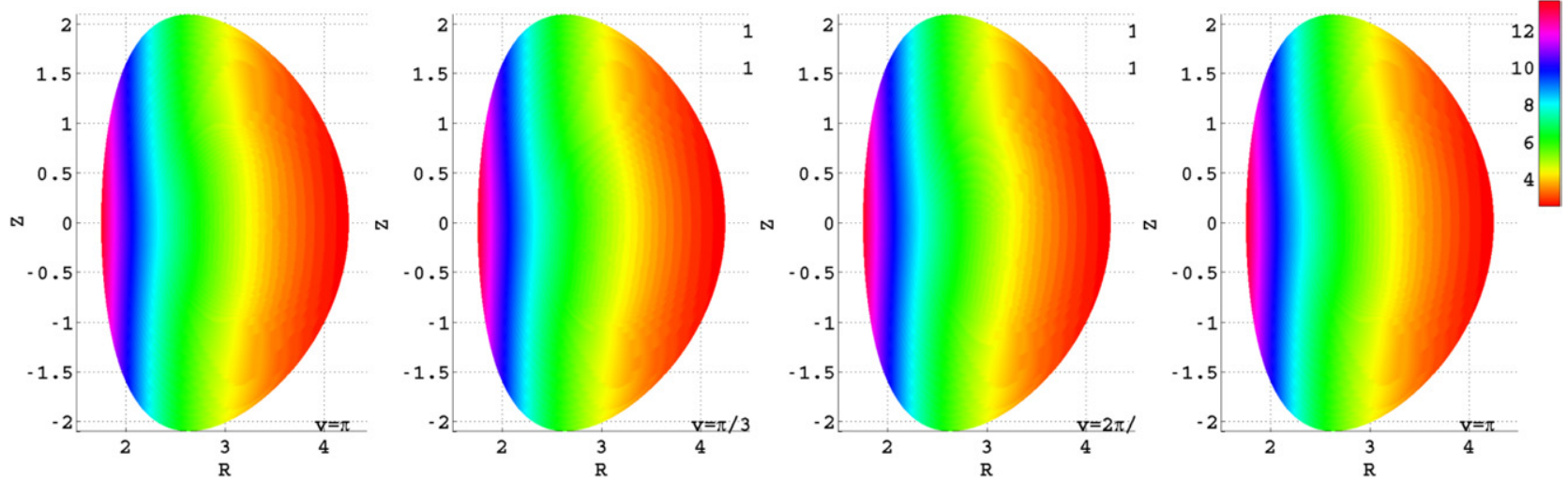

Figure 6. The distribution of constant plasma energy functional $\mu_{0} p+B^{2} / 2$ at 4 toroidal cross sections that span half the way around the torus at toroidal angles $v=0, v=\pi / 3, v=2 \pi / 3$ and $v=\pi$.

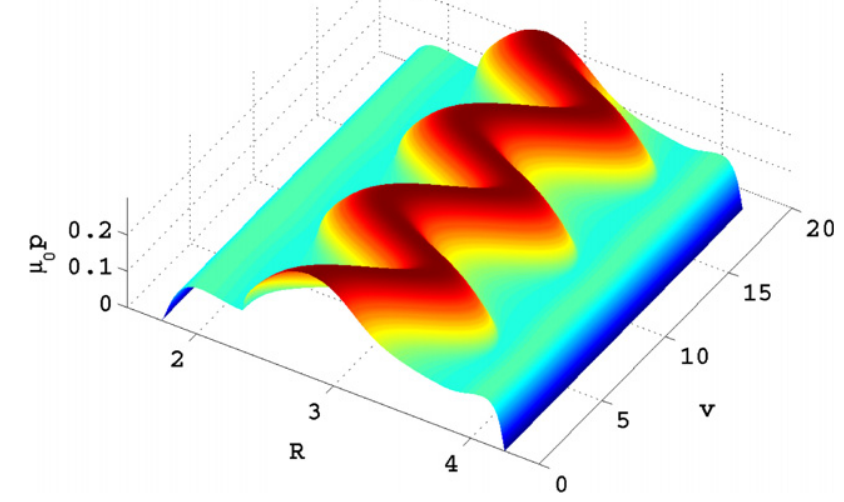

Figure 7. The normalized pressure $\mu_{0} p$ as a function of $R$ and the toroidal angle $v$ reveals the typical picture of a snake structure. In this reference equilibrium state $q_{\mathrm{min}}=1.004$.

yields a sequence of equilibria with $q_{\text {min }}$ descending from 1.03 to 0.94 with displaced helical core snake structures. In effect, a bifurcated set of equilibrium solutions are obtained. The initial conditions are identical for each case except for the guess of the position of the magnetic axis. If a sufficient helical distortion is chosen, the helical 3D snake solution is triggered. Otherwise, the axisymmetric solution branch is found. A comparison of

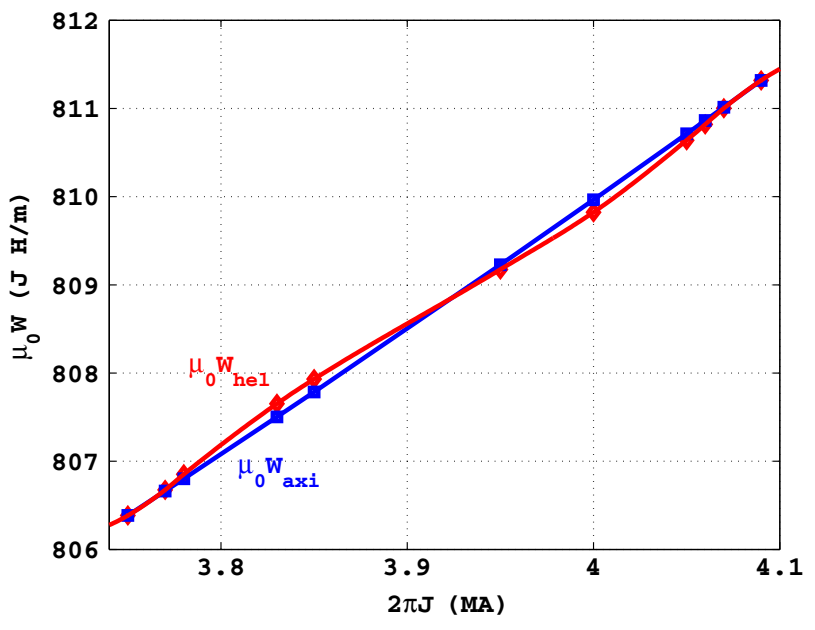

Figure 8. The equilibrium energy $\mu_{0} W$ for the bifurcated helical and axisymmetric branches as a function of the total toroidal current $2 \pi J$.

the internal energy $\mu_{0} W$ as a function of the toroidal current for the two solutions is presented in figure 8 which shows that the difference only corresponds to a small fraction of a per cent. At high current ( $4 \mathrm{MA})$, the snake-like helical branch has slightly lower energy. This corresponds to cases with $q_{\min }<1$. When $q_{\min }>1$, the axisymmetric branch 


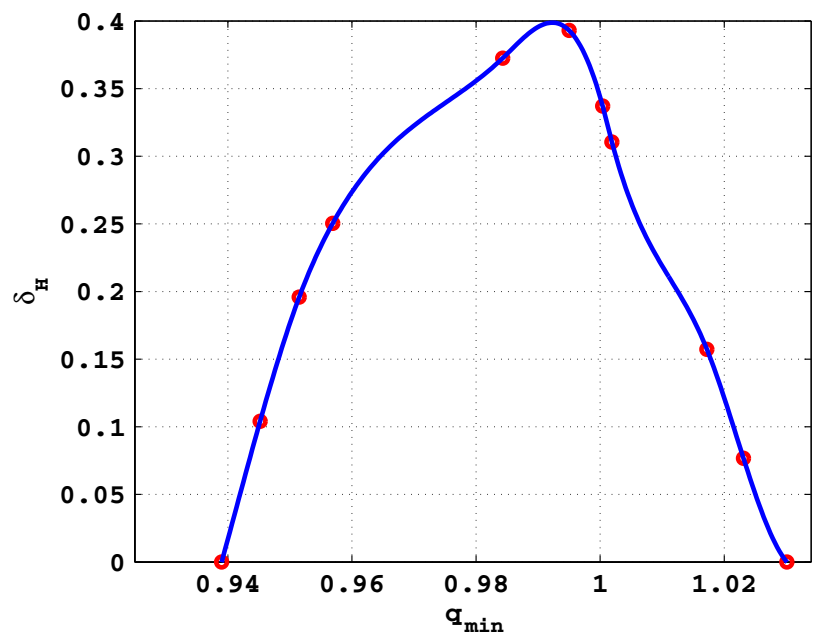

Figure 9. The magnetic axis helical distorsion parameter $\delta_{\mathrm{H}}$ as a function of $q_{\mathrm{min}}$, which corresponds to the minimal value of the inverse rotational transform.

has slightly lower energy. Nevertheless, we would like to emphasize that the difference is so minuscule that we cannot categorically state that one or the other constitutes a favoured equilibrium solution. To compare the results obtained further, we define a parameter that describes the helical deformation of the magnetic axis as

$$
\delta_{\mathrm{H}}=\frac{\sqrt{R_{01}^{2}(s=0)+Z_{01}^{2}(s=0)}}{a},
$$

where $R_{01}\left(Z_{01}\right)$ corresponds to the ( $\left.m=0, n=1\right)$ Fourier amplitude of $R(Z)$ at the magnetic axis and $a=1.61 \mathrm{~m}$ is the effective plasma minor radius. We plot $\delta_{\mathrm{H}}$ as a function of $q_{\min }$ in figure 9 . The reference equilibrium state with $3.85 \mathrm{MA}$ toroidal current we have previously described corresponds to the data point with $q_{\min }$ closest to unity. Each point on the curve in figure 9 represents an equilibrium state with a snakelike internal structure. A very relevant feature of the model we propose is that changes within the range $0.94<q_{\min }<1.03$ throughout a discharge could alter the size of the snake, but it would still persist. In the experimental discharges, sawteeth crashes are observed that shrink the size of the snake without suppressing it $[1,2]$. The $q=1$ island must survive a sawtooth event according to the standard model of the snake [3]. The predictions of our model, on the other hand, are much more robust inasmuch as they allow $q_{\min }$ to rise almost to 1.03 before the $3 \mathrm{D}$ helical deformations vanish. This constitutes a very clear and defining distinction between the two descriptions of the snake development. Furthermore, we conjecture that small oscillations in the $q$-profile within a discharge can lead to successive neighbouring equilibrium states described with our model in which the dimensions/amplitudes of the snake would appear to fluctuate. The conditions of a growing snake [2,3] can also be adequately handled with the model we propose if we envision that $q_{\text {min }}$ slides slowly towards unity either from above or below.

Internal ideal MHD kink modes can be nonresonantly unstable in an axisymmetric circular cross section tokamak with weak reversed central shear and $0<q_{\min }-1<0.014$ [18]. We verify that the axisymmetric branch of the sequence

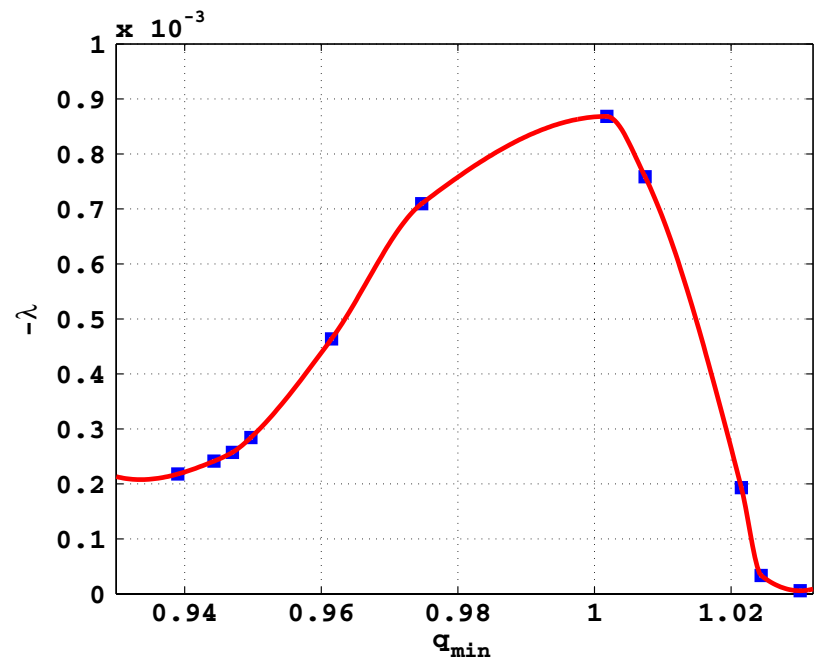

Figure 10. The magnitude of the ideal MHD internal kink eigenvalue $\lambda=-\omega^{2}$ as a function of $q_{\min }$ in the axisymmetric branch of the JET equilibrium configurations. The mode is dominantly $m=1, n=1$ ( $m(n)$ is the poloidal (toroidal) mode number). The last points $\left(q_{\min } \sim 1.025\right)$ coincide with the existence of a helical state in figure 9.

of configurations we have investigated in figure 9 is linearly unstable to the internal kink mode using the TERPSICHORE code $[19,20]$. Crucially, we find that the point of marginal stability with respect to the dominant $m=1, n=1$ mode in the axisymmetric equilibrium state aligns very closely with the point of vanishing helical excursion of the magnetic axis of the equilibrium calculation near $q_{\min } \simeq 1.03$ as shown in figure 10. This is consistent with the stabilization of the quasi-interchange mode when $q_{\text {min }}$ becomes sufficiently large [21]. The most unstable axisymmetric equilibrium occurs for $q_{\min }$ near unity. At the lower end of the range, with respect to $q_{\mathrm{min}}$, in which we compute snake equilibria with ANIMEC, the TERPSICHORE code predicts linear unstable ideal MHD modes but with significantly reduced magnitudes, consistent with the persistence of a classical internal kink mode under such conditions [21]. This constitutes the basis of our conjecture that the snake could be the result of the saturated state of this mode which could become the novel helical equilibria we have identified.

We have successfully computed MHD equilibrium states that reproduce snake-like structures for a JET tokamak configuration with an imposed axisymmetric boundary. The equilibrium is characterized by an internal 3D helical core with features similar to a saturated ideal $m=1, n=1$ internal kink mode. Central magnetic shear reversal with a minimum value of $q$ near unity is a critical aspect in the generation of the helical equilibrium. At $\langle\beta\rangle=2.3 \%$, equilibrium snake structures are computed in the range $0.94<$ $q_{\min }<1.03$. The axisymmetric branches of the JET equilibria in this range are unstable to linear ideal MHD internal $m=1,=1$ kink modes. The domain of finite helical axis excursion in the bifurcated equilibrium states coincides with that of linear ideal internal kink instability in the axisymmetric equilibrium states. The energy differential between the bifurcated snake and axisymmetric solutions is too small to definitively suggest a preferred equilibrium state. 
The standard theoretical description that a magnetic island formed about the $q=1$ rational surface is a necessary feature of a snake appears not to be a strict qualifying condition. Although it is possible that magnetic islands could be linked to the development of the snake, we believe that it would be difficult to distinguish experimentally the internal ideal kink-like equilibrium structure we compute from a $q=1$ island. In addition, the existence of such helical equilibria, with a snake-like structure, could explain why the snake, once formed, is very robust and hardly affected by sawtooth crashes for example [1,2].

A free boundary equilibrium description could facilitate the calculations of 3D helical structures by liberating the axisymmetric constraint imposed on the exact shape of the plasma. The condition of nested magnetic flux surfaces in our model preclude the generation of equilibrium states with magnetic islands. Saturated tearing modes could be investigated with more general, yet more computationally intensive and time consuming, codes [18, 22-27].

The authors greatly appreciate the contributions of Dr S.P. Hirshman to the development of the 3D equilibrium solvers we have applied. They are indebted for the suggestions of an anonymous referee to highlight the differences between this work and the standard description of the snake that has considerably improved this article. They would also like to thank Professor Francis Troyon for his support and encouragement of this research. This investigation was partially sponsored by the Fonds National Suisse de la Recherche Scientifique and by Euratom. The views and opinions expressed herein do not necessarily reflect those of the European Commission

(c) Euratom 2011.

\section{References}

[1] Weller A. et al 1987 Phys. Rev. Lett. 592303

[2] Gill R.D. et al 1992 Nucl. Fusion 32723

[3] Wesson J.A. 1995 Plasma Phys. Control. Fusion 37 A337

[4] Hender T.C. et al 2002 Plasma Phys Control. Fusion 441143

[5] Garabedian P. 2006 Proc. Natl Acad. Sci. USA 10319232

[6] Cooper W.A., Graves J.P., Pochelon A., Sauter O. and Villard L. 2010 Phys. Rev. Lett. 105035003

[7] Cooper W.A. et al 2011 Plasma Phys. Control. Fusion 53074008

[8] Reimerdes H. et al 2006 Plasma Phys. Control. Fusion 481621

[9] Camenen Y. et al 2007 Nucl. Fusion 47586

[10] Chapman I.T. et al 2010 Nucl. Fusion 50045007

[11] Menard J.E. et al 2005 Nucl. Fusion 45539

[12] Lazarus E.A. et al 2006 Plasma Phys. Control. Fusion 48 L65

[13] Cooper W.A., Graves J.P. and Sauter O. 2011 Plasma Phys. Control. Fusion 53024002

[14] Hirshman S.P. and Whitson J.C. 1983 Phys. Fluids 263553

[15] Hirshman S.P. and Betancourt O. 1991 J. Comput. Phys. 9699

[16] Cooper W.A. et al 2009 Comput. Phys. Commun. 1801524

[17] Grad H. 1966 Phys. Fluids 9498

[18] Aydemir A.Y. 1987 Phys. Rev. Lett. 59649

[19] Anderson D.V., Cooper W.A., Gruber R., Merazzi S. and Schwenn U. 1990 Int. J. Supercomput. Appl. 434

[20] Cooper W.A. 1992 Plasma Phys. Control. Fusion 341011

[21] Wesson J.A. 1986 Plasma Phys. Control. Fusion 28243

[22] Reiman A. and Pomphrey N. 1991 J. Comput. Phys. 94225

[23] Harafuji K., Hayashi T. and Sato T. 1989 J. Comput. Phys. 81169

[24] Hirshman S.P., Sanchez R. and Cook C. 2011 Phys. Plasmas at press

[25] Hudson S.R., Hole M.J. and Dewar R.L. 2007 Phys. Plasmas 14052505

[26] Lütjens H. and Luciani J.F. 2008 J. Comput. Phys. 2276944

[27] Halpern F.D., Leblond D., Lütjens H. and Luciani J.F. 2011 Plasma Phys. Control. Fusion 53015011 